\title{
The structural insight of class III of polyhydroxyalkanoate synthase from Bacillus sp. PSA10 as revealed by in silico analysis
}

\author{
Listia Pradani ${ }^{1}$, M. Saifur Rohman ${ }^{1,2,}$, Sebastian Margino ${ }^{2}$ \\ ${ }^{1}$ Graduate Program in Biotechnology, Graduate School of Universitas Gadjah Mada, Jl. Teknika Utara, Yogyakarta, 55281, Indonesia \\ ${ }^{2}$ Laboratory of Agricultural Microbiology, Department of Agricultural Microbiology, Faculty of Agriculture, Universitas Gadjah Mada, \\ Jl. Flora, Kompleks Bulaksumur UGM, Yogyakarta, 55281, Indonesia \\ ${ }^{*}$ Corresponding author: saifur@ugm.ac.id
}

SUBMITTED 13 September 2019 REVISED 22 January 2020 ACCEPTED 22 March 2020

\begin{abstract}
PhaC synthase is an enzyme responsible for PHA polymerization. In this work, the catalytic mechanism class III of PhaC synthase from Bacillus sp. PSA10 (BacPhaCSynt) was reported through in silico modelling approach based on the primary sequence of the PhaC synthase. The open reading frame BacPhaCSynt has been successfully isolated, cloned and overexpressed the recombinant protein in Escherichia coli BL21(DE3). To know the global architecture and catalytic mechanism, the structural prediction of BacPhaCSynt has been carried out by using MODELLER. The recombinant BacPhaCSynt exhibited monomeric molecular weight (MW) of $43.6 \mathrm{kDa}$, when it was analyzed on $12 \%$ SDS-PAGE gel. Based on the structural prediction, BacPhaCSynt exhibited global architecture of $\alpha / \beta$ hydrolase fold, with the root mean square deviation (r.m.s.d) value of $0.94 \AA$. The catalytic residues composition of BacPhaCSynt consists of C151, D307, and $\mathrm{H} 336$, but the $\mathrm{H} 336$ and D307 residues of the model have been distorted $62.8^{\circ}$ and $175.2^{\circ}$ from the corresponding residues of the template. Since the D307 is quite a distance from the H336, it might act as a general base for the activation of -OH group of the substrate. The results strongly suggested that the mode of action of BacPhaCSynt obeyed the covalent catalysis mechanism.
\end{abstract}

KEYWORDS polyhydroxyalkanoate (PHA); class III of PhaC synthase; a/ $\beta$ hydrolase; Bacillus sp. PSA10; MODELLER

\section{Introduction}

Nowadays, plastic is indispensable material that can be used in immense fields of application, such as packaging materials, parts in the electronics and automotive industry, and biomedical devices, etc. Despite the high impact of the plastics in our daily life, plastics also can cause severe the environmental problems. The growing accumulation of waste consisting of non-degradable carbon backbone plastic polymer, elevated $\mathrm{CO}_{2}$ and increasing toxic level in the atmosphere caused by the plastic incineration, are the most visible example of the negative impact of the plastic usage (Braunegg et al. 2004; Iwata 2015). Therefore, an alternative way to replace petroleum-based plastic is necessary. A bio-based material with plastic-like properties is regarded as the one alternative to overcome such a petroleum-based plastics usage problem. Bio-based material with plastic-like properties is more eco-friendly to the environment because of its biodegradability. Bio-based material is non-fossil-based; therefore it does not face the ongoing depletion of fossil resources.

Polyhydroxyalkanoates (PHA) is the one bio-based polymer that can be used as an alternative material to replace the petroleum-based plastics. Polyhydroxyalkanoates (PHA) are natural polyester comprising various hydroxylalkanoates (HAs) that have been considered as a feasible substitute to conventional petroleum-based plastics and elastomers (Sudesh et al. 2000; Zhang et al. 2009; Chen and Patel 2012; Chek et al. 2017). Various microorganisms have synthesized PHA as an energy and redox storage material typically under nutrient-limited conditions in the presence of an excess of carbon source $(\mathrm{Hu}$ et al. 2005). The PHA from Bacillus sp., Cupriavidus necator (formerly Ralstonia eutropha) and Pseudomonas sp. have been studied profoundly. However, the lack of understanding on PHA biosynthesis and the weak properties of the polymer, bacterial PHA has not been able to completely replace the petroleum-based polymer (Wittenborn et al. 2016; Sagong et al. 2018). Therefore, a complete understanding of the PHA biosynthesis is required for the sake of its application to replace the petroleumbased polymer.

PHA is synthesized by the reaction three enzymes, which consist of phaC synthase (PhaC)(EC.2.3.1.-), acetocaetyl Co-A 3-ketotiolase (PhaA)(EC. 2.3.1.9), and acetoacetyl Co-A reductase (PhaB)(EC.1.1.1.36) (Sagong 
et al. 2018). Among of them, PhaC synthase is the most important enzyme, since it is responsible for PHA polymerization (Chek et al. 2019). Based on their kinetic properties and catalytic mechanism, PhaC synthases are classified into four classes. The grouping of the PhaC synthase into each class is dependent on its structure of PhaC alone or in association with other subunit and substrate specificity: Class I, III, and IV produce short-chain length polymer depending on 3hydroxypropionate (3HP), 3-hydroxy or 4-hydroxy butyrate (3HB, 4HB), 3-hydroxyvalerate (3HV), and 3hydroxyhexanoate $(3 \mathrm{HH})$ precursors, while class II produce medium chain length of polymer depending on the 3hydroxyhexanoate (3HH), 3-hydroxyhepatanoate (3HHp), 3-hydroxyocatanoate (3HO), 3-hydroxy decanoate (3HD), 3-hydroxyundecanoate (3HUD), 3-hydroxydodecanoate (3HDD) (C6 to C12), and availability of the corresponding CoA thioester substrates, originating from three different metabolic pathways (Mezolla et al. 2018).

Class I and II are formed by a single protein, with a molecular weight of $60 \mathrm{kDa}$, but enzymatically active in homodimeric form. Each monomer consists of two domains, $\mathrm{N}$ domain which is located at the end of the $\mathrm{N}$ terminus and catalytic domain. The $\mathrm{N}$ domain functions for protein-protein interaction during dimerization processes. Class I and II also contain the lid or cap which has an important role for open and closing the substrate entrance gate (Chek et al. 2017).

In contrast to class I and II, class III and IV are composed of the two different protein subunits. The class III and IV of PhaC synthases requires the PhaE and PhaR subunit, respectively. In the case of class III of PhaC synthase, PhaE requires for PHA polymerization. Class IV of PhaC synthase is prevalent for the Bacillus group and as mention before that the protein is composed of the PhaC synthase and PhaR as an accessory subunit. The PhaC synthase subunit, with a molecular weight of $40 \mathrm{kDa}$, of class IV, contains PhaC box sequence ([GS]-X-C-X-[GA]-G) that located at the catalytic core. PhaR of the subunit composition of class IV of PhaC synthase has a molecular weight of $20 \mathrm{kDa}$ which is comparable to the archaeal-type PhaEs. It seems to be that the PhaR also functions for PHA polymerization since the PhaC synthase alone shows enzymatic activity. PhaR has an additional function for alcoholysis mechanism (Tsuge et al. 2015; Kihara et al. 2017). Alcoholysis mechanism is important for the regulation of the PHA molecular weight and also for modifying of PHA terminus. The amino acids sequence identity between the class III and IV of PhaC synthase subunit is 34 percent, which is considered as high enough homology, by the fact that among the members of class III of PhaC synthase have an identity of 38 percent (Tsuge et al. 2015).

Regarding the PhaC synthase catalytic mechanism, a various mechanism has been proposed. One mechanism is referred to as a non-processive ping-pong model. The ping-pong model requires two cysteine residues for monomer chain transfer during the polymer elongation. In order to perform such a mechanism, the PhaC synthase must be in dimeric form. The second mechanism is the processive model. The processive model does not necessarily require two cysteine residues. Therefore, the processive model can be taken place in a single catalytic site (Mezolla et al. 2018). The third mechanism that has been proposed based on the $C$. necator PhaC synthase crystal structure implies that once newly 3HB-CoA enters the catalytic core, it would be nucleophile attacked by cysteine residue to produce 3HB-Cys. Following the binding of incoming substrate (3HB)2CoA, the hydroxyl group of 3HB-Cys is deprotonated by the histidine residue, facilitated through basicity modulation by the aspartate residue (Wittenborn et al. 2016). All the proposed catalytic mechanisms are based on the crystal structure of class I of PhaC synthases that have been recently determined. For example, the PhaC synthase from $C$. necator, a class I of PhaC synthase, has been determined from two independent groups (Wittenborn et al. 2016; Kim et al. 2017). Another class I of PHA synthase came from Chromobacterium USM2 has also been determined (Chek et al. 2017). It remains elusive though, how the PhaC synthases perform their catalytic mechanism, as well as the class III of PhaC synthase. To the best of our knowledge, there is no crystal structure either from class II, III and IV have been reported.

Recently we have isolated the PhaC synthase encoding gene from Bacillus sp. PSA10. BlastX result indicated that the PhaC synthase from Bacillus sp. PAS10 (BacPhaCSynt) belongs to the class III PhaC synthase. Although class I and class III produce a similar type of PHA polymer, class III requires accessory subunit (PhaE) for its catalysis (Liebergesell and Steinbuchel 1992; Müh et al. 1999). The results showed that the global architecture of BacPhaCSynt was similar to the class I PhaC synthase, as well as the catalytic mechanism. Interestingly, the analysis also observed that Bacillus sp. PSA10 has PhaR instead of PhaE, in which both proteins have lower homology. Taken together of the finding, it might suggest that class III and IV of PhaC synthase are similar enzymes, but they have different accessories subunit.

\section{Materials and Methods}

\subsection{Bacterial cell and Polyhydroxyalkanoate (PHA) ac- cumulation test}

Bacillus sp. PSA10 (previously isolated from the waste of sago starch processing) was used in this experiment (Yanti et al. 2009). To confirm whether the bacterial isolate able to produce PHA, Bacillus sp. PSA10 was examined for its ability to produce polyhydroxyalkanoate (PHA) polymer on Ramsay minimal media containing $2 \%$ of soluble starch as a carbon source. The bacterial cell was cultivated at room temperature for 24 h (Berger et al. 1989; Ramsay et al. 1990). The PHA accumulation was examined after $24 \mathrm{~h}$ of cultivation by a light microscope and transmission electron microscope (TEM) (Mesquita et al. 2015). 


\subsection{Bacterial cell and genome preparation}

For the genomic preparation, Bacillus sp. PSA10 was cultivated in Luria-Bertani (LB) media for $48 \mathrm{~h}$ at room temperature. After that, cell was harvested by centrifugation at $8000 \mathrm{x}$ g for $5 \mathrm{~min}$. The cell pellet was collected and further subjected for genomic isolation. Bacterial genome isolation was carried out using the Wizard Genomic DNA Extraction Kit (Promega). All the genomic DNA isolation followed the manufacture's recommendations. The obtaining genomic DNA was then stored in $-20^{\circ} \mathrm{C}$ for further analysis.

\subsection{PhaC synthase open reading frame isolation and cloning}

To amplify the class III of PhaC synthase and PhaR open reading frame (orf) from Bacillus sp. PSA10, the following pairs of primer was designed base on the PhaC synthase orf sequences from Bacillus group (Accession no. KU233683.1, AB525784 for phaC and phaR, respectively). The sequence of the primes were phbC_f: 5-ATGGCAATTCCTTACGTGCAAGAG3, phbC_r: 5-TTATTTAGAGCGTTTTTCTAGCC-3, phaR_f: 5-ATGGAACAGCAAAAAGTATTTGATCC-3 and phaR_r : 5-TTACTTGCGAGCTGGCTGCTC-3 for the forward and reverse primers, respectively. For the cloning purpose of PhaC synthase orf, the same sequence of the primers was used, except the addition of recognition site for $\mathrm{NcoI}$ and EcoI. Therefore, the sequence of the primers were phbC_f_NcoI: 5ATATATCCATGGCAATTCCTTACGTGCAAGAG-3, phbC_r_EcoRI: 5-ATATATATGAATTCTTATTTAGA GCGTTTTTCTAGCC-3. Underlined letters indicate the recognition sites for NcoI and EcoRI, respectively. Poly- merase chain reaction (PCR) was performed with T100 ThermoCycler (BioRad) using KOD Polymerase (Toyobo) according to the procedures recommended by the supplier. All DNA oligomers for PCR were synthesized by Macrogen. The DNA sequence was carried out by 1st BASE to further confirm whether the amplified product was the open reading frame of PhbC synthase. To clone the orf of PhaC synthase, the orf was re-amplified by PCR using a pair of primers carrying the recognition site for NcoI and EcoRI. The PCR product was then purified by GeneHplow (Geneaid) and then subjected for DNA digestion. Fast Digest NcoI and EcoRI (Thermo Scientific) restriction enzymes were employed for this purpose. For the expression vector, pET26a (Novagen) was used in this experiment. pET26a vector was linearized by double digestion with the same restriction enzymes. Ligation of the DNA fragment into the expression vector was carried out using Fast DNA ligation kit. The ligation product was named as pET-BacPhaCSynt hereafter, and used to transform E. coli BL21(DE3) (Novagen). The growing colonies were then subjected for colony PCR using the T7 promoter and $\mathrm{T} 7$ terminator primers (Macrogen) to select the positive clones.

\subsection{Overexpression of recombinant BacPhaCSynt}

To examine the expression of the recombinant PhaC synthase, the E. coli BL21(DE3) carrying recombinant pETBacPhaCSynt, was grown in LB media supplemented with $50 \mathrm{mg} / \mathrm{mL}$ of kanamycin, at $37^{\circ} \mathrm{C}$ for $48 \mathrm{~h}$. On the following day, the culture was transferred into fresh LB media containing $50 \mathrm{mg} / \mathrm{mL}$ of kanamycin and the growth was continued for $2 \mathrm{~h}$ until the optical density (OD600) of the culture reached 0.5 . The culture was then induced by 1 $\mathrm{mM}$ of isoprophylthio- $\beta$-D-galactopyranoside (IPTG) for

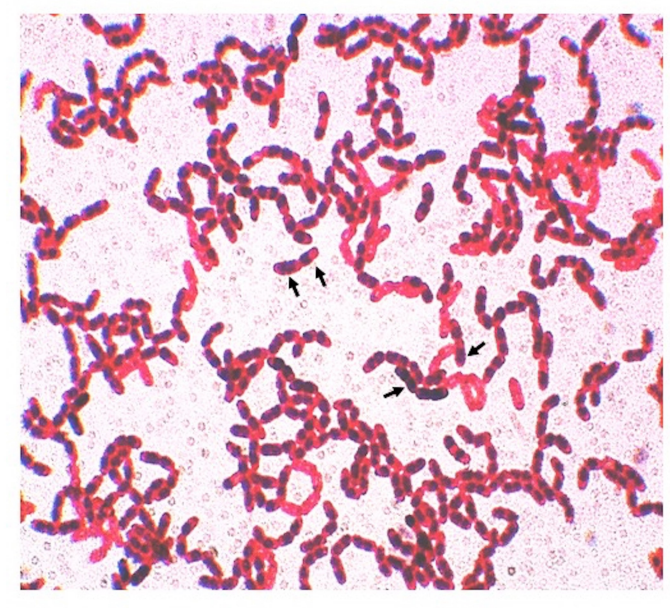

(a)

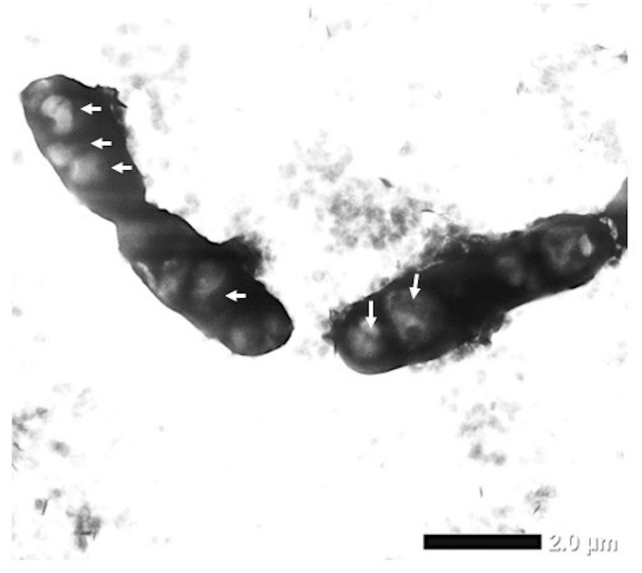

(b)

FIGURE 1 Polyhydroxy alkanoat (PHA) accumulation in Bacillus sp. PSA10. The PHA accumulation was examined under the (a) light and (b) transmission electron microscope (TEM). In order to examine the PHA accumulation under light microscopy Sudan Black staining was employed, PHA accumulation in the cells was indicated by the mass with black color in the cell (black arrows). PHA accumulation examined under TEM was shown as the mass with white color (white arrows). Black solid bar is equal to $2 \mu \mathrm{m}$. 
an additional $3 \mathrm{~h}$. After $3 \mathrm{~h}$ the $5 \mathrm{~mL}$ cell culture was then harvested by centrifugation at $8000 \mathrm{x}$ g for $5 \mathrm{~min}$. The cell pellet was collected and subjected for SDS-PAGE analysis (Laemmli 1970).

\subsection{Phylogenetic tree construction}

To analyze the evolutionary history of PhaC synthase, the phylogenetic tree of all class $\mathrm{PhaC}$ synthase was constructed. A total of 46 amino acid sequences of PhaC synthase were used for the phylogenetic construction. Among 46 amino acid sequences, one was the amino acid sequence of BacPhaCSynt. The phylogenetic tree was constructed by using the MEGA7 suite. The evolutionary history of the tree was inferred by using the Neighbor-Joining method (Saitou and Nei 1987) and the evolutionary distance was computed using the Poisson correction method (Zuckerkandl and Pauling 1965). To test the integrity of the tree, 500 replicates of the bootstrapping was employed.

\subsection{Modeling class III BacPhaCSynt}

Class III of BacPhaCSynt was modeled by using the MODELLER v.9.20 suite (Webb and Sali 2016). ITASSER suite was used to find the most appropriate template of the model (https://zhanglab.ccmb.med.umich.edu/

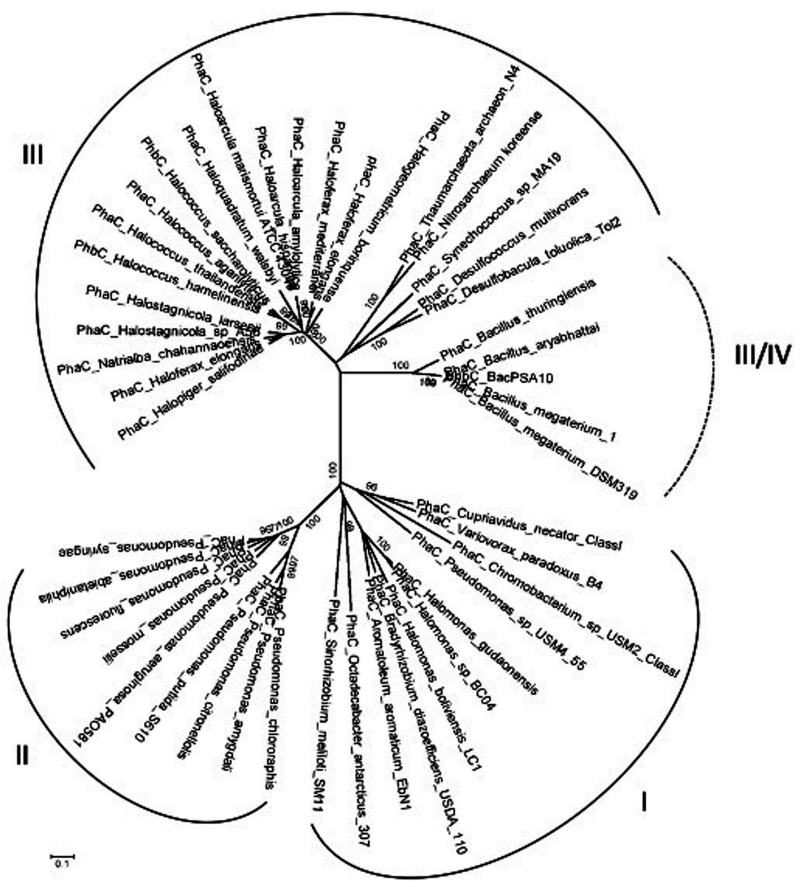

FIGURE 2 Evolutionary relationships of PhaC synthases from bacteria and archaeal.The evolutionary history was inferred using the Neighbor-Joining method (Saitou and Nei 1987) The optimal tree with the sum of branch length $=9.19428895$ is shown. The tree is drawn to scale, with branch lengths in the same units as those of the evolutionary distances used to infer the phylogenetic tree. The evolutionary distances were computed using the Poisson correction method (Zuckerkandl and Pauling 1965) are in the units of the number of amino acid substitutions per site. The analysis involved 46 amino acid sequences. All positions containing gaps and missing data were eliminated. There were a total of 251 positions in the final dataset. Evolutionary analyses were conducted in MEGA7 (Kumar et al. 2016).
I-TASSER/) (Yang and Zhang 2015; Yang et al. 2015). ITASSER analysis produces several appropriate templates to model the BacPhaCSynt. The best template for modeling of BacPhaCSynt was class I of PhaC synthase structure (PDB accession no. 5t6o) from $C$. necator with the identity value of 27 percent. The five models of BacPhaCSynt generated by MODELLER was then selected for the best model based on the lowest value of molpdf and DOPE (Webb and Sali 2016). The selected model was then further refined by running on the ModRefiner (https://zhangl ab.ccmb.med.umich.edu/ModRefiner/) (Yang and Zhang 2015; Yang et al. 2015) to produce the protein structure model with the best physical quality of the local structure. The model structure was refined iteratively and stopped when the best structure model was obtained. Every refined product was then evaluated to their stereochemical quality of the model by analyzing residue by residue and overall structural geometry by using SAVES v.5.0 suite program (https://servicesn.mbi.ucla.edu/SAVES/) (Morris et al. 1992; Hooft et al. 1996). The best quality of the model would be expected to have over 90 percent of residues in the most favored regions. PyMol Version 2.1.1 was used to visualize the model (PyMol), measurement of the residue-residue distance, residue-substrate distance, and torsion angle. PyMol also used for manipulation of the substrate analog docking. To be noted that the energy binding value of the docking result was not calculated. The CAVER 3.0.3 plugin suite was employed to predict the possible substrate tunnel of the model. Standard parameters of the tunnel prediction were as follows: minimum probe radius was set at $0.9 \AA$; shell depth and shell radii were 4 and $3 \AA$, respectively; clustering threshold was set to $3.5 \AA$; starting point used was all the structure fold and the starting point for optimization was set for maximum distance was set to $3 \AA$ and the desired radius was set to 5Å (Chovancová et al. 2012).

\section{Results and Discussion}

\subsection{Cloning and expression of class III of BacPhaCsynt open reading frame}

Previously it has been reported that Bacillus sp. PSA10 could accumulate the polyhydroxyalkanoate (PHA) polymer (Yanti et al. 2009). To further confirm the previous result, the ability of the Bacillus sp. PSA10 to accumulate PHA was tested. The bacterial cell was grown in Ramsay minimal medium containing $2 \%$ of soluble starch. The PHA accumulation was examined microscopically (light microscope and TEM) after $24 \mathrm{~h}$ cultivation. The result confirmed that Bacillus sp. PSA10 showed an ability to accumulate PHA with the soluble starch as a carbon source (Fig. 1a and b). Previously, it has been reported that the PHA type accumulated by Bacillus sp. PSA10 was polyhydroxybutyrate (PHB)(Yanti et al. 2009). This further suggested that Bacillus sp. PSA10 might have PhaCSynt encoding gene.

To confirm whether Bacillus sp. PSA10 genome har- 


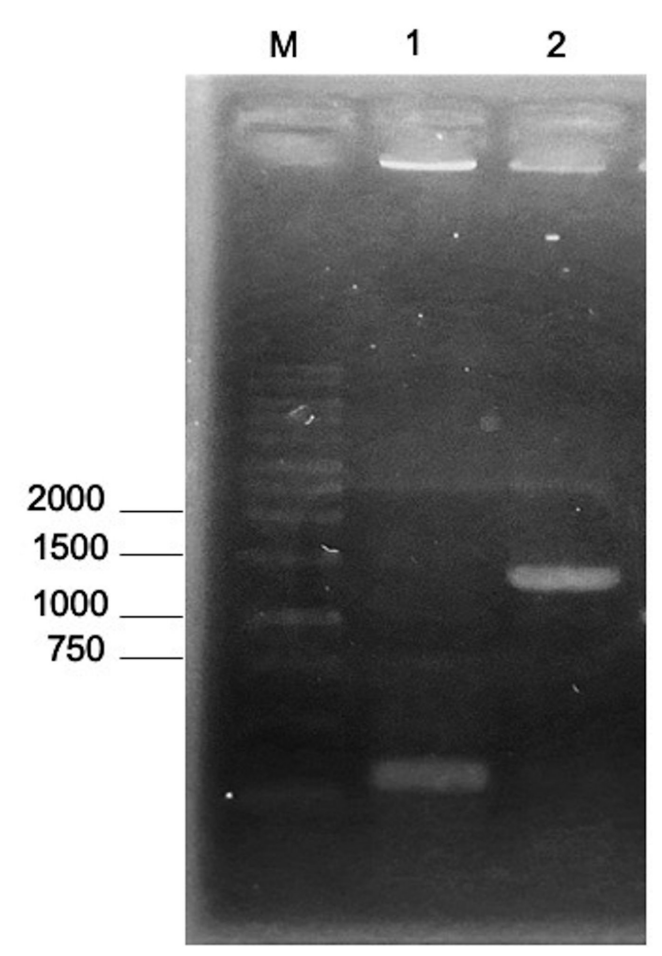

FIGURE 3 Cloning of BacPhaCSynt open reading frame into expression vector ( $\mathrm{pETSystem}$ ). Selection for positive clone was carried out by colony PCR method. T7 promoter and T7 terminator primers were employed for colony PCR. M. 1kb DNA ladder, Lane 1. Negative control; Lane 2. Positive clone.

bor the genes necessary for PHA biosynthesis, the open reading frame (orf) of PhaC synthase have been amplified by using a pair of primers that were designed base on class III of PhaC synthase from Bacillus group. By those primers, the open reading frame of class III of PhaC synthase from Bacillus sp. PSA10 has been successfully amplified (data not shown). BlastX analysis indicated that the sequence of class III of PhaC from Bacillus sp, PSA10 (Accession no. MT312251) was highly homolog to the class III of PhaC synthase from Bacillus megaterium DSM319 with the identity of $98.9 \%$. However, phylogenetic tree analysis indicated that class III and IV of PhaC synthase showed a close relationship (Figure 2). As shown in Figure 2, the PhaC from Bacillus aryabhathai dan Bacillus megaterium_1 belong to the class IV of PhaC synthase, meanwhile, the PhaC synthase of Bacilllus megaterium DSM319 and Bacillus thuringiensis belongs to the class III group. The bootstrap value of $\mathrm{PhaC}$ synthase Bacillus sp. PSA10 and Bacillus megaterium_1, the value was 34\%. Meanwhile, the bootstrap value between the PhaC synthase of Bacillus sp. PSA10 and Bacillus megaterium DSM319 was 89\%. These results suggested that the BacPhaCSynt belongs to the class III of PhaC synthase. However, scanning on the Bacillus megaterium DSM 319 genome, only PhaR was present, but not PhaE, which was the subunit composition of class III of PhaC synthase. To confirm whether Bacillus sp. PSA10 genome contains the PhaR, the open reading frame of PhaR was amplified. The open reading frame of PhaR from the Bacillus

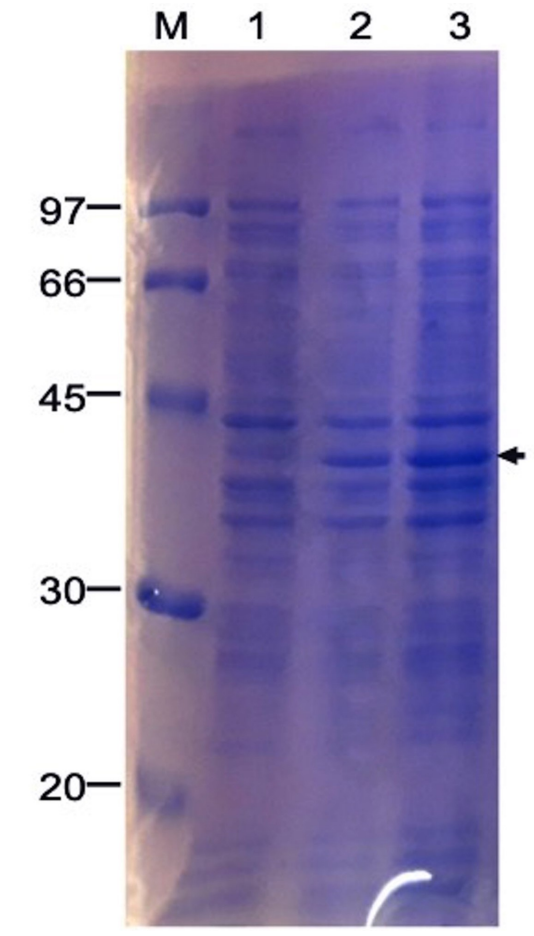

FIGURE 4 SDS-PAGE analysis of the BacPhaCSynt expression. BacPhaCSynt expression was analyzed on $12 \%$ SDS-PAGE and stained with Comassie Brilliant Blue staining. M: LMW marker, Lane 1. Un-induced; Lane 2. Induced for 1 hour; Lane 3. Induced for 2 hours. Arrow indicates the expressed recombinant protein.

sp. PSA10 genome was successfully amplified (data not shown). BlastX result of the nucleotide sequence of the PhaR of Bacillus sp. PSA10 (Accession no. MT312252) exhibited high homology to the PhaR of Bacillus group with an identity of $96 \%$ to $97 \%$ (Fig. 1S, Supplementary material). This result might suggest that the class III and class IV of PhaC synthase were the same enzymes but different the accessories subunit requirement. The highest homology of class III and class IV of PhaC synthase further confirmed our prediction. Moreover, class III and class IV of PhaC synthase both contain Abhydrolase_1, without any additional domain (Liu et al. 2019).

To examine whether the open reading frame of class III of PhaC synthase from Bacillus sp. PSA10 could produce recombinant protein, the open reading frame of BacPhaCSynt was cloned in an expression vector and used to transform E. coli BL21(DE3) as a host. Following the positive cloned confirmation (Figure 3 ), the positive cloned was further examined for BacPhaCSynt recombinant protein expression. Since the expression is under control of lac promoter, the isoprophyl thio- $\beta$-D-galactopyranoside (IPTG) was used to induce the expression of the recombinant class III of BacPhaCSynt. The recombinant protein was induced after $2 \mathrm{~h}$ of incubation with IPTG and prolonged the incubation time to another 1 hour did not significantly increase the expression level (Figure 4). Disruption of cell suspension in $10 \mathrm{mM}$ Tris-HCl bufffer $\mathrm{pH} 8.0$ by sonication indicated that the cell suspension turbidity did not turn into translucent, indicating that the recombi- 


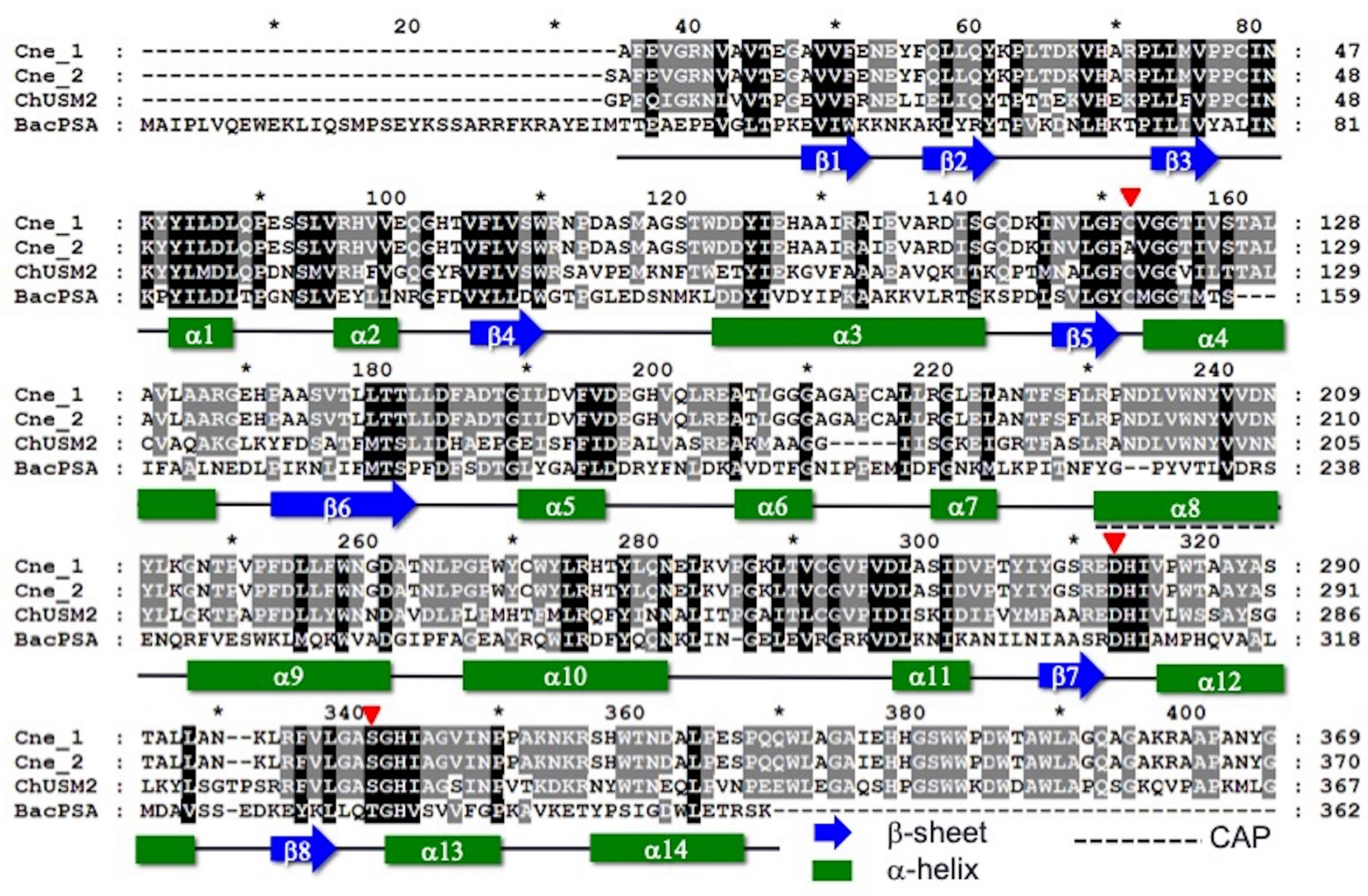

FIGURE 5 Amino acid sequences alignment of BacPhaCSynt (BacPSA) (Accession no. MT312251) with class I of PhaC synthase of C. necator (Cne1 and Cne2)(Accession no. AEl76811) and Chromobacterium USM2 (ChUSM2)(Accession no. ADL70203.1). Predicted secondary structure of BacPhaCSynt is also shown. Red inverted triangle indicates the location of catalytic residues.

nant BacPhaCSynt formed inclusion body (Pradani, personal communication). Previously, it was also reported that overexpression of PhaC synthase from Chromatium vinosum under the control of T7lac promoter produced only $25 \%$ of solubility (Müh et al. 1999). The insolubility of the recombinant protein indicates that the recombinant protein might not properly fold. Several proteins require a chaperone to help their proper folding. It is already known that the class III of PhaC synthase requires another subunit (PhaE or PhaR) for its activity. Our recombinant protein may require the presence of PhaE or PhaR to present in a soluble form. Therefore, it is noteworthy to construct the co-expression system to simultaneously overexpress the PhaC synthase and PhaE or PhaR subunit.

\subsection{The global fold class III of BacPhaCSynt}

To understand the catalytic mechanism, in silico modelling of class III of BacPhaCSynt has been carried out. According to the best of our knowledge, the only class I of PhaC synthase structure is available, there is no report regarding the structure of other classes of PhaC synthase. Class I, III, and IV produce similar polyhydroxyalkanoate polymer depending on the type of precursor, however, the catalytic mechanism of class III remains elusive. Unlike the class I PhaC synthase, class III requires another subunit that is encoded by a different gene (phaE) (Liebergesell and
Steinbuchel 1992; Müh et al. 1999). To model the class III of BacPhaCSynt, the orf of class III of PhaC synthase Bacillus sp. PSA10 was translated into the amino acid sequence. Based on the sequence alignment, BacPhaCSynt showed the sequence identity of $18 \%$ to $27 \%$ to the class I of PhaC synthase. All the catalytic residues of BacPhaCSynt are located on the fully conserved region. Secondary prediction indicates that BacPhaCSynt contains 14 of $\alpha$ helix and 8 of $\beta$-sheet (Figure 5).

To predict the 3D structure of BacPhaCSynt, the amino acid sequence was then used to find the appropriate template to model the structure. By running the I-TASSER suite, it was found that the most appropriate template to model the class III of BacPhaCSynt was the structure class I of PhaC synthase (PhaCCn) from C. necator (PDB accession no. $5 t 6 o A$ ) with the identity of $27 \%$ which is in agreement with the amino acid sequence alignment. The I-TASSER result was then used to model the BacPhaCSynt by using the MODELLER. From the modelling results, 5 models of BacPhaCSynt were constructed and among them, model 1 (phbC.B99990001.pdb)(Table 1S, Supplementary material) was the best, with the molpdf and DOPE scores were 2146.21 and -39167.52, respectively. The resulting model was then refined by using the crystal structure of class I of $C$. necator (PDB accession no. 5hz2). After several times refinement of the model, the most appro- 


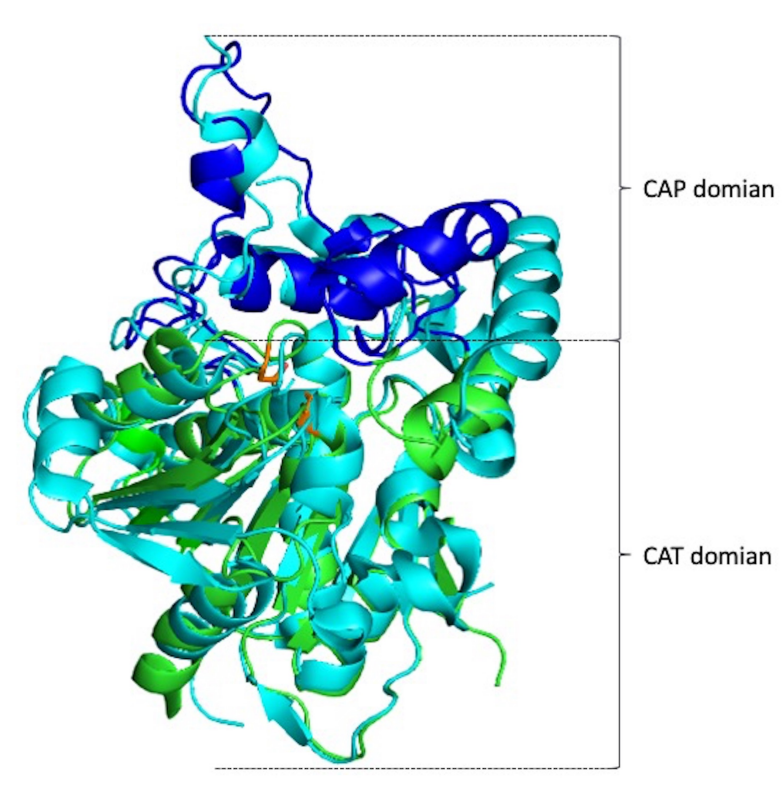

FIGURE 6 The superposition between the model of BacPhaCSynt and template. Model and template are shown in green and cyan, respectively. Both model and template showed the root mean square deviation (r.m.s.d) value of 0.94A. The fold shows the two domains, CAT domain and CAP domain. Catalytic residues are located in the CAT domain as indicated by orange color. CAP domain is shown in blue.

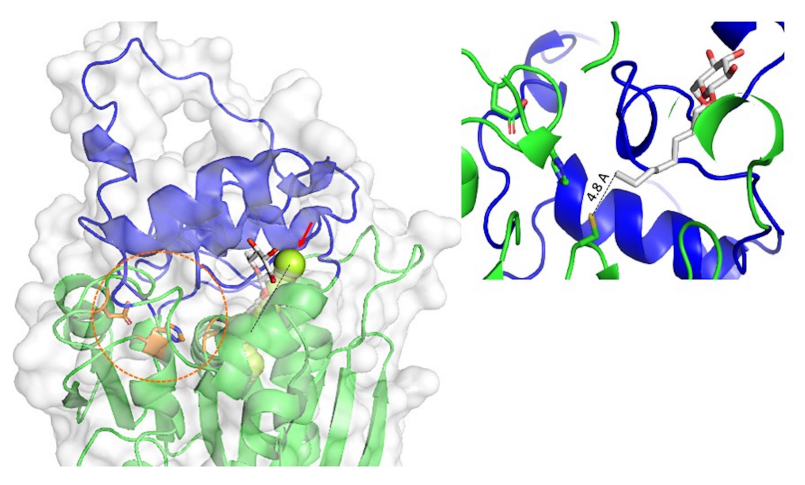

FIGURE 7 The substrate tunnel entrance of BacPhaCSynt shows the substrate entrance mechanism. The green spares represent the tunnel diameter and the red arrow indicates the entrance site. The substrate analog $\beta$-octyl glucoside ( $\beta$-OG)(grey) is also shown. Black dashed line indicates the tunnel path. The orange circle indicates the catalytic core location. Inset indicates the distance of the $\beta$-OG to the C151 residue. The distance was $4.8 \AA$.

priate model was obtained with over $90 \%$ of the amino acid residues in the most favoured regions of the Ramachandran plot (Fig. 2S, Supplementary material). The resulting model has a similar fold to the template with the root mean square deviation (r.m.s.d) value of $0.94 \AA$. The model of the BacPhaCSynt has $\alpha / \beta$ fold which is the common super secondary structure of the $\alpha / \beta$ hydrolase family (Figure 6 ). The structural model of BacPhaCSynt also contains CAP and CAT domains. The CAP domain functions for closing and opening the substrate channel. Open and close mechanism of the CAP domain important for the regulation of the accessing of the catalytic center. When the CAP do-

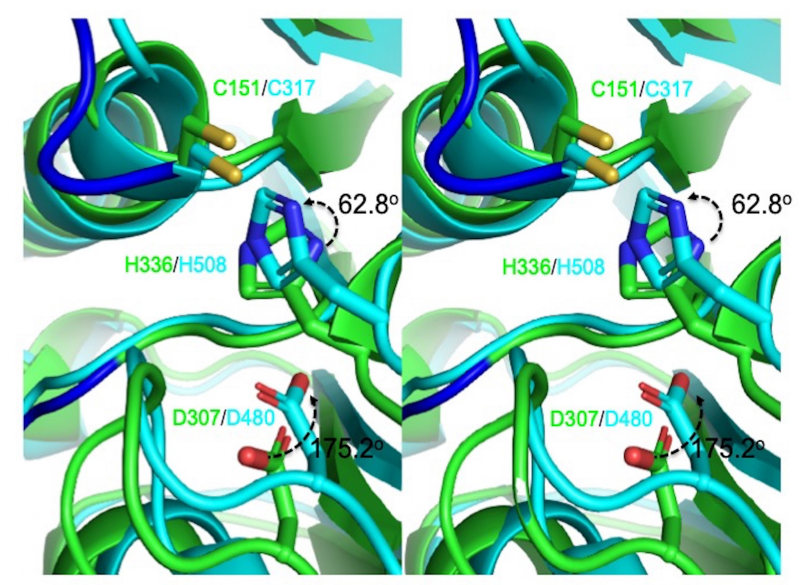

FIGURE 8 The catalytic residue orientation of BacPhaCSynt in compared to the class I of PhaC synthase. The catalytic residues of the BacPhaCSynt composed from C151; D307 and H336. The distortion of the $\mathrm{D} 307\left(175.2^{\circ}\right)$ and $\mathrm{H} 336\left(62.8^{\circ}\right)$ residues orientation compared to its template is shown. Black curve dashed arrows indicate the degree of distortion.

main flips away, it will open the entrance of the catalytic center that makes product release and substrate entrance from or to the catalytic center (Mezolla et al. 2018; Chek et al. 2017).

In this work, the prediction had been focused on the single subunit of the protein, since the single subunit was sufficient to explain the global fold of the protein. Although, the biological activity of class I of PhaC synthase is in homodimeric form, the symmetrical dimeric architecture in which the active site of each monomer is separated from one another by $33 \AA$ across an extensive dimer interface, suggesting that the catalytic mechanism occurs in single subunit (Wittenborn et al. 2016).

\subsection{Substrate entrance channel of BacPhaCSynt}

By using the model of BacPhaCSynt, the substrate channel pattern of the model structure was predicted by employing the CAVER ver. 3.0.3 suite. The substrate channel was located in proximity to the CAP domain (Figure 7). When the substrate analog $\beta$-octyl glucoside ( $\beta$-OG) was fitted onto the predicted channel, it clearly showed that the substrate analog was fit to the path of the channel (as indicated the black dashed line, Figure 7) The end of the substrate analog was also directed into the catalytic core. The end of the substrate analog seems to be approaching to the cysteine residue (C151). The distance of the end of the substrate analog to the C151 was $4.8 \AA$. Such distance, however, is quite far for the donor-acceptor proton process. Therefore, the $\beta$-OG is only to show the path of the substrate entry, not for showing the nucleophile attack by the C151 residue (see possible catalytic mechanism). Substrate analog of $\beta$-OG is the inhibitor that is commonly used for co-crystallization of lipase to make the lid of lipase performs in the open conformation (Roussel et al. 2002). 


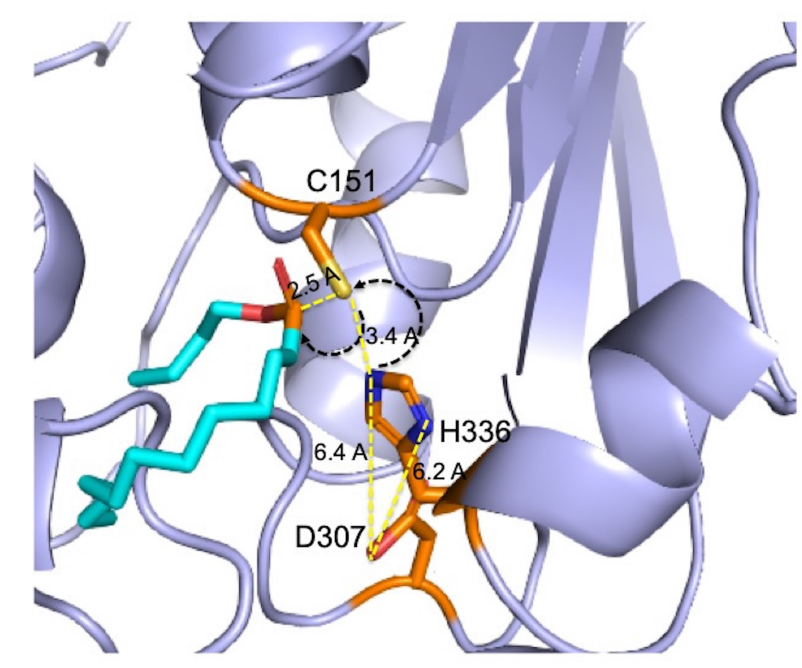

FIGURE 9 The possible interaction between substrate analog and catalytic residues of BacPhaCSynt. Substrate analog was C11Y4 phosphonate, an inhibitor of the dog gastric lipase (DGL). The distance of $C 151$ residue is only $2.5 \AA$ from the ester bond of the substrate. The distance of $\mathrm{C} 151$ and $\mathrm{H} 336$ residue is $3.4 \AA$ (yellow

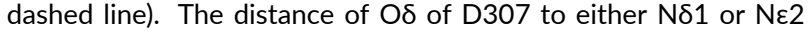
of $\mathrm{H} 336$ is $6.2 \AA$ and $6.4 \AA$ (yellow dashed line), respectively. Black curve dashed arrows indicate the electron transfer.

\subsection{Possible catalytic mechanism of BacPhaCSynt}

By the model, the orientation of the catalytic residues of class III BacPhaCSynt could be determined. The catalytic residues of the class III BacPhaCSynt were cysteine (C151), aspartate (D307) and histidine (H336), which were similar to the catalytic triad of class I of PhaCCn synthase. The catalytic residues of model class III of BacPhaCSynt have a similar orientation to that of class I of PhaCCn synthase (Figure 8). However, the residue D307 and H336 showed little distortion from the corresponding residues of the template. The D307 distorts $175.2^{\circ}$ from the configuration of D480, while H336 distorts $62.8^{\circ}$ from the configuration of H508. Despite the distortion orientation of the D307 and H336, the presentation of all these three residues suggested that BacPhaCSynt had a similar catalytic mechanism to the class I of PhaC synthase. When the substrate analog of C11Y4 phosphonate was docked on to the catalytic core of the BacPhaCSynt, the ester bond of the substrate is close enough to the $\mathrm{C} 151$ residue, which is only $2.5 \AA$ (Figure 9). Within this distance, it makes possible for the C151 residue to do nucleophile attacks the ester bond of the substrate analog. The atomic distance within 2.2-2.5 $\AA$ is categorized as a strong electrostatic interaction (Jeffrey 1997). The Nع1 of H336, which is only $3.4 \AA$ from the C151, makes H336 possible to performs hydrogen atom abstraction of sulfhydryl (-SH) group of C151 residue, although such distance is categorized as the weak electrostatic. From figure 9, it also clearly showed that the position of D307 quite far from the H336. The distance

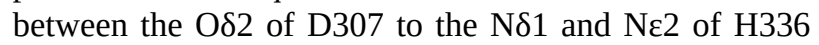
was 6.2 and $6.4 \AA$, respectively, which is quite far for the deprotonation of H336 by the D307 residue. Therefore, the D307 is not part of the catalytic triad but functions as a general base catalyst in the activation of the 3-OH group of HB-CoA (Tian et al. 2005).

\section{Conclusions}

In this work the open reading frame of BacPhaCSynt, a class III of PhaC synthase from Bacillus sp. PSA10 was successfully isolated and overexpressed the recombinant protein in the E. coli system. In silico analysis clearly showed that the global fold of the BacPhaCSynt was similar to the class I of PhaC synthase with the r.m.s.d value of $0.97 \AA$. The model structure of BacPhaCSynt also suggested that class III of PhaC synthase has a similar catalytic mechanism to that of class I of PhaC synthase, specifically it obeys the covalent catalysis mechanism. Therefore, our results give a hints the catalytic mechanism of class III PhaC synthase.

\section{Supplementary Materials}

Figure 1S. Alignment of PhaR from Bacillus sp. PAS10 with its homolog; Tabel 1S. Five model generated by MODELLER based on the template class I polyhydroxy alkanoate synthase C. necator (5t6o); Figure 2S. Ramachandran plot of the refined model BacPhaCSynt.

\section{Acknowledgments}

This work has been supported by the grant in aid for Final Project Recognition (RTA) of Universitas Gadjah Mada with the contract No. 2129/UN1/DITLIT/DITLIT/LT/2019.

\section{Authors' contributions}

LP carried out the laboratory work and data collection. MSR conceived of the study, analyzed the data and wrote the manuscript; SM provides the material for the research work. All authors read and approved the final version of the manuscript.

\section{Competing interests}

The authors declare no competing interest.

\section{References}

Berger E, Ramsay BA, Ramsay JA, Chavarie C, Braunegg G. 1989. PHB recovery by hypochlorite digestion of non-PHB biomass. Biotechnol Tech. 3(4):227-232. doi:10.1007/BF01876053.

Braunegg G, Bona R, Koller M. 2004. Sustainable polymer production. Polym Plast Technol Eng. 43(6):1779-1793. doi:10.1081/PPT-200040130. 
Chek MF, Hiroe A, Hakoshima T, Sudesh K, Taguchi S. 2019. PHA synthase (PhaC): interpreting the functions of bioplastic-producing enzyme from a structural perspective. Appl. Microbiol. Biotechnol. 103(3):1131-1141. doi:10.1007/s00253-018-9538-8.

Chek MF, Kim SY, Mori T, Arsad H, Samian MR, Sudesh K, Hakoshima T. 2017. Structure of polyhydroxyalkanoate (PHA) synthase PhaC from Chromobacterium sp. USM2, producing biodegradable plastics. Sci Rep. 7(1):1-15. doi:10.1038/s41598-017-055094.

Chen GQ, Patel MK. 2012. Plastics derived from biological sources: present and future: a technical and environmental review. Chem Rev. 112(4):2082-2099. doi:10.1021/cr200162d.

Chovancová E, Pavelka A, Benes P, Strnad O, Brezovsky J, Kozlikova B, Gora A, Sustr V, Klvana $\mathrm{M}$, Medek $\mathrm{P}$, Biedermannová L, Sochor J, Damborský J. 2012. CAVER 3.0: a tool for the analysis of transport pathways in dynamic protein structures. PLoS Comput Biol. 8(10):e1002708. doi:10.1371/journal.pcbi.1002708.

Hooft RW, Vriend G, Sander C, Abola EE. 1996. Errors in protein structure. Nature 381:272-272. doi:10.1038/381272a0.

Hu WF, Sin SN, Chua H, Yu PHF. 2005. Synthesis of polyhydroxyalkanoate (PHA) from excess activated sludge under various oxidation-reduction potentials (ORP) by using acetate and propionate as carbon sources. Appl Biochem Biotechnol. 121:289-301. doi:10.1385/ABAB:121:1-3:0289.

Iwata T. 2015. Biodegradable and bio-based polymers: future prospects of eco-friendly plastics. Angew Chem Int Ed. 54(11):3210-3215. doi:10.1002/anie.201410770.

Jeffrey GA. 1997. An introduction to hydrogen bonding, volume 12. New York: Oxford university press.

Kihara T, Hiroe A, Ishii HM, Mizuno K, Tsuge T. 2017. Bacillus cereus-type polyhydroxyalkanoate biosynthetic gene cluster contains R-specific enoyl-CoA hydratase gene. Biosci Biotechnol Biochem. 81(8):1627-1635. doi:10.1080/09168451.2017.1325314.

Kim J, Kim YJ, Choi SY, Lee SY, J KK. 2017. Crystal structure of Ralstonia eutropha polyhydroxyalkanoate synthase C-terminal domain and reaction mechanisms. Biotechnol J. 12(1):1600648. doi:10.1002/biot.201600648.

Kumar S, Stecher G, Tamura K. 2016. MEGA7: molecular evolutionary genetics analysis version 7.0 for bigger datasets. Mol Biol Evol. 33(7):1870-1874. doi:10.1093/molbev/msw054.

Laemmli UK. 1970. Cleavage of structural proteins during the assembly of the head of bacteriophage T4. Nature. 227(5259):680-685. doi:10.1038/227680a0.

Liebergesell M, Steinbuchel A. 1992. Cloning and nucleotide sequences of genes relevant for biosynthesis of poly(3-hydroxybutyric acid) in Chromatium vi- nosum strain D. Eur J Biochem. 209(1):135-150. doi:10.1111/j.1432-1033.1992.tb17270.x.

Liu Z, Zhu Z, Yang J, Wu S, Liu Q, Wang M, Cheng H, Yan J, L W. 2019. Domain-centric dissection and classification of prokaryotic poly (3-hydroxyalkanoate) synthases. bioRxiv p. 693432. doi:10.1101/693432.

Mesquita DP, Amaral AL, Leal C, Oehmen A, Reis MAM, Ferreira EC. 2015. Polyhydroxyalkanoate granules quantification in mixed microbial cultures using image analysis: Sudan Black B versus Nile Blue A staining. Anal Chim Acta. 865:8-15. doi:10.1016/j.aca.2015.01.018.

Mezolla V, D’Urso OF, Poltronieri P. 2018. Role of PhaC type I and type II enzymes during PHA biosynthesis. Polymers. 10(910):1-12. doi:10.3390/polym10080910.

Morris AL, MacArthur MW, Hutchinson EG, Thornton JM. 1992. Stereochemical quality of protein structure coordinates. Proteins: Struct Funct Bioinf. 12(4):345-364. doi:10.1002/prot.340120407.

Müh U, Sinskey AJ, Kirby DP, S LW, Stubbe JA. 1999. PHA synthase from Chromatium vinosum: cysteine 149 is involved in covalent catalysis. Biochemistry. 38(2):826-837. doi:10.1021/bi9818319.

Ramsay BA, Lomaliza K, Chavarie C, Dube B, Bataille P, Ramsay JA. 1990. Production of poly-(beta-hydroxybutyric-co-beta-hydroxyvaleric) acids. Appl Environ Microbiol. 56(7):2093-2098. doi:10.1128/AEM.56.7.2093-2098.

Roussel A, Miled N, Berti-Dupuis L, Rivière M, Spinelli S, Berna P, Gruber V, Verger R, Cambillau C. 2002. Crystal structure of the open form of dog gastric lipase in complex with a phosphonate inhibitor. J Biol Chem. 277(3):2266-2274. doi:10.1074/jbc.M109484200.

Sagong HY, Son HF, Choi SY, Lee SY, J KK. 2018. Structural insight into polyhydroxyalkanoates biosynthesis. Trends Biochem Sci. 43(10):790-805. doi:10.1016/j.tibs.2018.08.005.

Saitou N, Nei M. 1987. The neighbor-joining method: a new method for reconstructing phylogenetic trees. Mol Biol Evol. 4(4):406-425. doi:10.1093/oxfordjournals.molbev.a040454.

Sudesh K, Abe H, Doi Y. 2000. Synthesis, structure and properties of polyhydroxyalkanoates: biological polyesters. Prog Polym Sci. 25(10):1503-1555. doi:10.1016/S0079-6700(00)00035-6.

Tian J, Sinskey AJ, Stubbe JA. 2005. Detection of intermediates from the polymerization reaction catalyzed by a D302A mutant of class III polyhydroxyalkanoate (PHA) synthase. Biochem. 44(5):1495-1503. doi:10.1021/bi047734z.

Tsuge T, Hyakutake M, Mizuno K. 2015. Class IV polyhydroxyalkanoate (PHA) synthases and PHA-producing Bacillus. Appl Microbiol Biotechnol. 99(15):62316240. doi:10.1007/s00253-015-6777-9.

Webb B, Sali A. 2016. Comparative protein structure modeling using MODELLER. Curr Protoc Bioinf. 
54(1):5-6. doi:10.1002/cpbi.3.

Wittenborn EC, Jost M, Wei Y, A SJ, Drennan CL. 2016. Structure of the catalytic domain of the class I polyhydroxybutyrate synthase from Cupriavidus necator. J Biol Chem. 291(48):25264-25277. doi:10.1074/jbc.M116.756833.

Yang J, Yan R, Roy A, Xu D, J P, Zhang Y. 2015. The ITASSER Suite: protein structure and function prediction. Nat. Methods 12(1):7. doi:10.1038/nmeth.3213.

Yang J, Zhang Y. 2015. I-TASSER server: new development for protein structure and function predictions. Nucleic Acids Res. 43(W1):W174-W181. doi:doi.org/10.1093/nar/gkv342.

Yanti NA, Sembiring L, S M. 2009. Production of Poly- $\alpha$ hydroxybutyrate (PHB) from Sago Starch by The Native Isolate Bacillus megaterium PSA10. IJ Biotech. 11(1):1111-1116. doi:10.22146/ijbiotech.7804.

Zhang X, Luo R, Wang Z, Deng Y, Q CG. 2009. Application of (R)-3-hydroxyalkanoate methyl esters derived from microbial polyhydroxyalkanoates as novel biofuels. Biomacromol. 10(4):707-711. doi:10.1021/bm801424e.

Zuckerkandl E, Pauling L. 1965. Evolutionary divergence and convergence in proteins. In: V Bryson, HJ Vogel, editors, Evolving genes and proteins. Amsterdam: Elsevier. p. 97-166. doi:10.1016/B978-1-4832-27344.50017-6. 